\title{
Smooth inductively coupled ring trap for atoms
}

\author{
P. F. Griffin, E. Riis, and A. S. Arnold \\ Department of Physics, SUPA, University of Strathclyde, Glasgow G4 ONG, United Kingdom
}

(Received 6 March 2008; published 13 May 2008)

\begin{abstract}
We propose and numerically investigate a scalable ring trap for cold atoms that surmounts problems of roughness of the potential and end effects of trap wires. A stable trapping potential is formed about an electrically isolated, conducting loop in an ac magnetic field by time averaging the superposition of the external and induced magnetic fields. We investigate the use of additional fields to eliminate Majorana spin-flip losses and to create a stable trapping geometry. The possibility of microfabrication of these ring traps offers the prospect of developing Sagnac atom interferometry in atom-chip devices.
\end{abstract}

DOI: $10.1103 /$ PhysRevA.77.051402

PACS number(s): 37.10.Gh, 37.25.+k, 67.85.-d

There is significant interest among the cold-atom community to build devices utilizing the sensitivity of matter-wave optics. This research is driven not only by the desire to explore new regimes of atomic physics, but also by the possibility of building sensors based on matter-wave interferometry [1,2]. An extremely promising method of achieving these goals is through microfabricated structures for trapping and manipulating atoms on atom chips [3,4]. While trapping and manipulation of atoms near permanent magnetic structures has been an active research topic for a number of years [5], much current work is focused on electromagnetic trapping as this allows for dynamic control of the trapping potentials. With modest currents, on the order of $1 \mathrm{~A}$, large magnetic field gradients and curvatures can be obtained in close proximity to the wires creating the magnetic potentials. Furthermore, due to the use of modern semiconductor fabrication techniques, complex structures can be constructed [6]. One example of this was the demonstration of the controlled transport of atomic clouds along an atom chip "conveyor belt" [7].

A leading motivation behind atom chip experiments is the desire to explore reduced dimensional quantum gasses [8]. However, fragmentation of atomic clouds close to trapping wires has been observed by many groups [9-12], which presents a significant problem for single-mode waveguiding of atoms and coherent matter-wave optics. In $[9,11]$, this roughness of the trapping potential was shown to be due to longitudinal magnetic fields arising from deviations of the current flow from the desired path. By recognizing that the roughness of the potential is proportional to the current through the trap wires, these defects were recently circumvented through the use of ac currents to generate the magnetic fields [13]. With this method, the corrugation of the trapping potential averages to zero while still maintaining the desired trap depths and frequencies.

A complementary approach to explore reduced dimensional quantum gases is ring traps. Even in the largest aspect ratio one-dimensional (1D) traps, there is finite confinement and extension along the "weak" trapping direction. In a ring trap, the weak direction has infinite extent with periodic boundary conditions. This opens the way for investigation of superfluid phenomena in quantum gases $[14,15]$. For sensor development, a symmetric ring potential is ideal for a Sagnac atom interferometer $[16,17]$ as the shared paths of opposing arms of the interferometer provide common mode rejection of noise due to path-length differences. To date, magnetic ring traps have involved the use of magnetic or electromagnetic elements to create a multiply connected magnetic field minimum in circular [16-19] and stadium [20] geometries. In these cases, electrical connectivity of the coils to an external power supply leads to a perturbation of the ideal symmetric field. When the traps are scaled down and the ring trap forms close to the current-carrying coils, these effects become increasingly significant.

Here we propose a scalable, smooth ring trap for ultracold atoms. No external electrical connection is required, eliminating undesirable end effects of wires and maintaining the symmetry of the waveguide. Instead, modest ac magnetic fields are applied to the system. We consider the simple system of a single, closed conducting loop formed from a conductor of circular cross section immersed in a magnetic field, the amplitude of which varies sinusoidally in time. The ac field is aligned perpendicular to the plane of the coil and is assumed spatially uniform across the area of the coil. From Faraday's law, we find that the time-varying $B$ field induces current in the conducting loop, creating an induced magnetic field about the loop. The external and induced fields cancel symmetrically in a ring, Fig. 1, the radius of which varies in time. If the potential varies at a frequency much greater than the atomic motional frequencies, then a single trapping radius is found by averaging the field over one cycle [18,21]. As the induced currents are inherently alternating, the potentials obtained will benefit from the suppression of roughness demonstrated in [13]. The ring trap forms in the plane of the

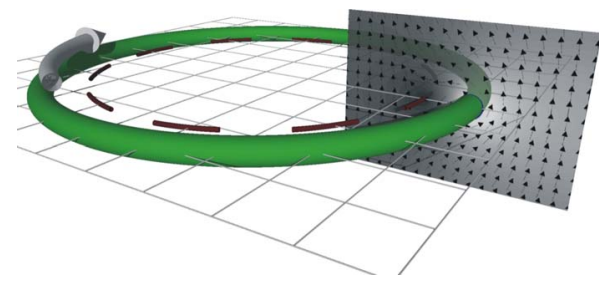

FIG. 1. (Color online) Schematic of the instantaneous vector fields for the system described in the text. The external bias field, increasing in magnitude, points upwards, while the induced current in the loop, indicated by the arrow, creates a field about the loop. The fields cancel at a radius inside the loop, indicated by the red, dashed circle. The gray scale in the field slice indicates field magnitude and arrows show the field direction. 

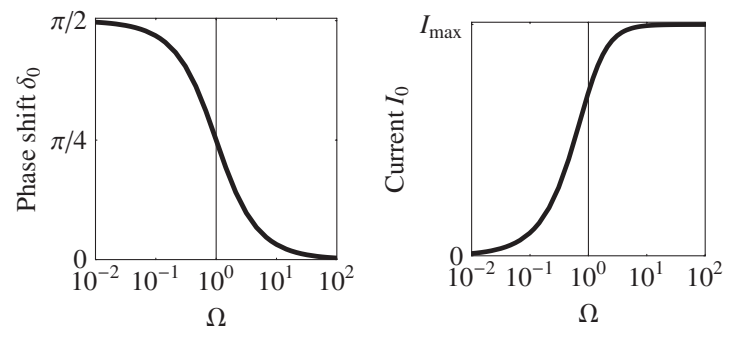

FIG. 2. The phase between current in the conducting loop and the driving field (left) and the induced current (right) as a function of the frequency of the driving field. Note that at low frequencies, the phase-induced current lags $\pi / 2$ behind the driving field. For the simulations in Fig. $3, \Omega=4$.

conducting loop and thus the zero of the instantaneous $B$ field travels through this trapping radius during each cycle, which may result in Majorana spin-flip losses of trapped atoms.

Elliptical integrals must be evaluated to obtain the field from a circular loop [22]. Initial investigations examined a full spatial integral across the wire for a uniform current density [23]. It was found that modeling the current as passing through an infinitesimal region centered on the wire yielded trapping parameters that agreed to better than $0.5 \%$ with the full model. For the simulations in this Rapid Communication, unless stated otherwise, we examine a loop of radius $r_{\text {ring }}=1 \mathrm{~cm}$ formed from gold wire of circular cross section, with a radius $r_{\text {wire }}=0.5 \mathrm{~mm}$.

The induced electromotive force, $\mathcal{E}$, in a loop is proportional to the rate of change of the magnetic flux, $\phi$, through the loop,

$$
\mathcal{E}=-\frac{\mathrm{d} \phi}{\mathrm{d} t}=L \frac{\mathrm{d} I}{\mathrm{~d} t}+I R
$$

For a magnetic field of the form $B(t)=B_{0} \cos (\omega t)$, the induced current, $I(t)$, may be expressed as

$$
I(t)=\frac{-I_{\max }}{\sqrt{1+\Omega^{-2}}} \cos \left(\omega t+\delta_{0}\right),
$$

where we have introduced the terms $I_{\max }=\pi r_{\text {ring }}^{2} B_{0} / L, \Omega$ $=\omega L / R$, and $\delta_{0}=\tan ^{-1}(1 / \Omega)$. The resistance and inductance of the ring are denoted by $R$ and $L$, respectively. Equation (2) applies once turn-on effects, such as overshooting of the current, have stabilized. The inductance of the loop is dependent on the loop radius, as well as the shape of the conductor forming the loop [24],

$$
L \approx \mu_{0} r_{\text {ring }}\left[\ln \left(8 r_{\text {ring }} / r_{\text {wire }}\right)-1.75\right] .
$$

The resistance is found from the familiar formula incorporating the resistivity, $\rho$, of the conductor,

$$
R=2 \rho r_{\text {ring }} / r_{\text {wire }}^{2} \text {. }
$$

Due to the resistance and inductance of the conducting loop, the phase and amplitude of the induced current are dependent on the frequency of the driving field, Fig. 2.

The trapping potential is obtained by time-averaging over one cycle of the varying fields. In Fig. 3(a), a contour
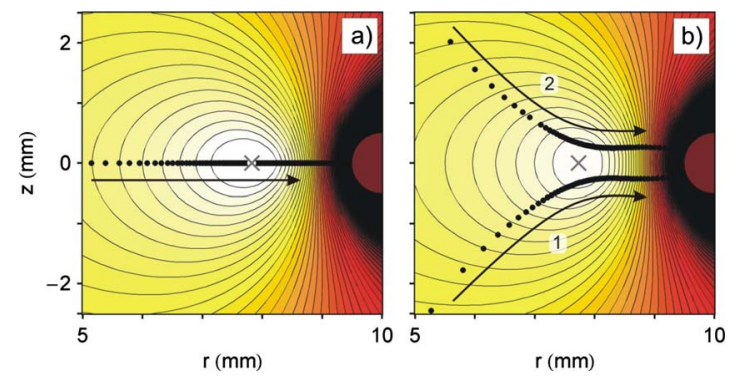

FIG. 3. (Color online) The magnetic potential about the conducting loop showing the effect of the control parameters. The positions of the time-averaged trap minima are marked by the gray crosses, and the instantaneous zeros of the $B$ field are denoted by the black dots. The trajectory that the minima follow is indicated by the numbered, black arrows. For these results, $\omega=(2 \pi) 30 \mathrm{kHz}$, $B_{0}=100 \mathrm{G}$. (a) No additional fields. (b) A dc quadrupole field, $B_{\mathrm{dcq}}=25 \mathrm{G} \mathrm{cm}^{-1}$, is added.

plot shows the potential in the radial-axial plane, with $B_{0}=100 \mathrm{G}$ and $\Omega=4$. However, the zeros of the time-varying field pass through the trapping radius once per cycle, which is undesirable for a magnetic atom trap. If we apply a dc quadrupole $B$ field, $B_{\text {dcq }}\{z,-r / 2\}$, centered on the conducting loop, we obtain the trapping potential shown in Fig. 3(b). The instantaneous zeros of the total $B$ field follow a trajectory that lies symmetrically about the plane of the ring, removing potential spin-flip losses. Furthermore, the form of the time-averaged potential is not significantly perturbed by the quadrupole field. The application of a quadrupole field with axial gradient $25 \mathrm{G} \mathrm{cm}^{-1}$ forms a stable ring trap $1.76 \mathrm{~mm}$ from the surface of the wire in the plane of the loop. For rubidium, trapping frequencies of 60 and $49 \mathrm{~Hz}$ radially and orthogonal to the plane of the loop, respectively, are obtained and a minimum trap depth of $1.9 \mathrm{mK}$, limited by gravity. For atom temperatures above $1.2 \mathrm{mK}$, the ring trap is no longer confined toward the center of the ring. Spin flip losses due to the orbiting trajectory of zero magnetic field yield an equilibrium temperature of $60 \mu \mathrm{K}$ in the trap, though this may be raised to $>200 \mu \mathrm{K}$ by increasing the amplitude of the quadrupole field. This feature may be used for evaporative cooling into the ring. However, the efficiency may be reduced due to the form of the trajectory of the field zeros. The average magnetic field at the trap radius is $18.8 \mathrm{G}$, and the minimum field at that point over one cycle is $9.7 \mathrm{G}$. As a result of the significant $B$ field at all times, the trap's Larmor frequency is typically two orders of magnitude larger than the rate of change of the magnetic field direction. Trapped atoms will thus adiabatically follow the magnetic field at all times, further preventing Majorana spin changes of trapped atoms [25].

The ring trap is conservative and as such must be loaded with cold atomic samples [26]. The use of a magnetic quadrupole field suggests that a mirror magneto-optical trap (mirror-MOT) [27] may be formed above the center of the loop, which may then be relaxed to load the ring trap. Precooled atoms may be transferred into the ring trap by spatially overlapping a separate atom trap, such as an optical tweezers [10]. Mode-matching of these potentials is likely to prove difficult and instead we propose a method of deform- 


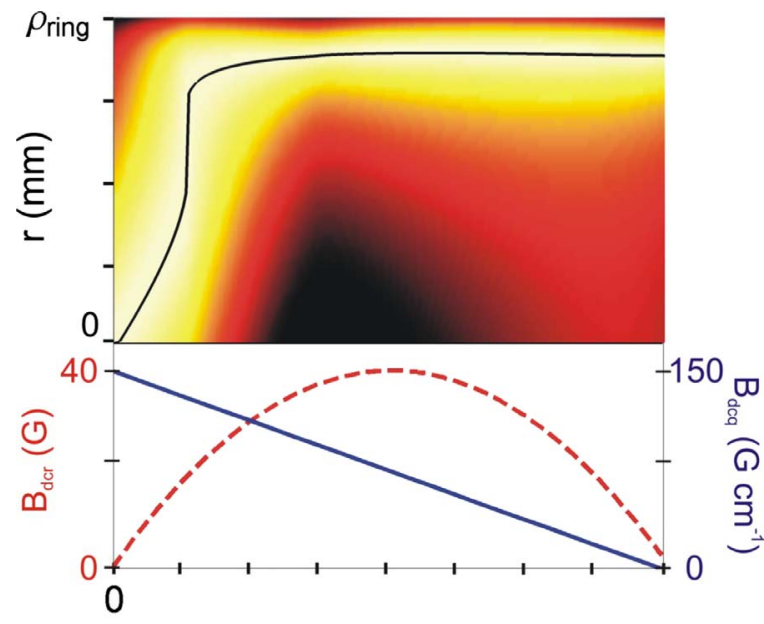

Time

FIG. 4. (Color online) The transfer from the single quadrupole potential to a ring trap. The quadrupole field is reduced while a transverse bias field is applied to shift the zero of the quadrupole toward the ring radius. The top figure shows the potential along a radius parallel to the bias field as the applied fields vary in time, shown in the lower figure.

ing the ring potential to provide a 3D magnetic trap. For large $B$-field gradients, the quadrupole field is the dominant term, forming a trap at the center of the ring. A bias $B$ field parallel to the ac driving field shifts the central quadratic trap out of the plane of the loop, providing improved optical access for initial loading of the potential with precooled atoms. An additional homogeneous bias field, $B_{\mathrm{dcr}}$, in the plane of the ring shifts the quadrupole trap toward the ring trap position. To localize atoms along the ring, and to minimize losses during the transfer, the quadrupole field is reduced and a bias field is gradually applied. The adiabatic nature of the transfer may be seen in Fig. 4 together with the applied fields. The time-averaging of the ac fields ensures that the instantaneous trap position is maintained at a nonzero $B$ field during the transfer except when the trap is at the ring center. Spin-flip losses at this point may be reduced by minimizing the duration the trap spends at this position or with additional timeaveraging fields. The weak bias $B$ field along the plane of the loop has the effect of tilting the ring and breaks the symmetry of the ring and may be used to localize atoms within the ring [19].

A maximum current density of $j=9300 \mathrm{~A} \mathrm{~cm}^{-2}$ is found for the parameters discussed, with corresponding Ohmic losses of $5.2 \mathrm{~W}$. The heating losses in the ring scale as $\left(B_{0}\right)^{2}$, whereas the trap frequencies scale as $\sqrt{B_{0}}$, allowing a significant reduction in the power dissipated, if required. These values offer promise for scaling the ring down, with the consideration of some additional parameters. First, a smaller loop necessarily has a smaller area, and thus couples less magnetic flux for the same $B$ field. Second, while the resistance varies as $r_{\text {ring }} / r_{\text {wire }}^{2}$, the inductance has a more complex scaling, Eq. (3). As an example, for a 2-mm-diam gold ring formed of wire with a diameter of $100 \mu \mathrm{m}$, in a $10 \mathrm{G}$ field at $1 \mathrm{MHz}$, we predict trapping frequencies of $(113,210) \mathrm{Hz}$. Notably, the current density in this case is the same as for the

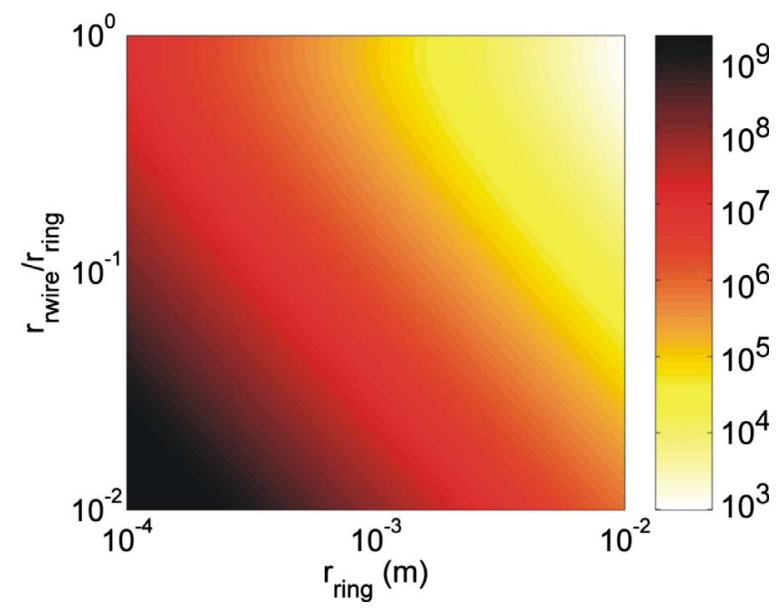

FIG. 5. (Color online) The scaling of drive frequency, $\omega_{\text {drive }}$ $\sim \omega=R / L$, as a function of $r_{\text {ring }}$ and $r_{\text {wire }}$. The $y$ axis is presented as the ratio of the length scales in the range of experimental interest.

larger ring, but the total dissipated power is three orders of magnitude lower. These parameters may be examined in conjunction by considering the frequency scaling $\omega_{0}=R / L$, which gives an order-of-magnitude frequency for the external ac field required to drive the system. Shown in Fig. 5, for ring radii below $\sim 100 \mu \mathrm{m}$, frequencies on the order of $100 \mathrm{MHz}$ are required to drive the system. The trap frequencies scale approximately inversely with the ring diameter for constant $B_{0}$, although a full simulation including all parameters discussed is required for accurate values. At the atom chip scale, the required quadrupole fields can be applied locally [28], allowing for parallelism of the device construction.

A further geometry considered is the extension to two concentric, coplanar conducting loops. The induced currents through each loop are related by their mutual inductance and Eq. (2) is extended to two coupled equations for these currents. For this system, the frequency of the external $B$ field should be chosen $\omega<R / L$ so that the separate coil currents run out of phase with the external field. In the limit of zero drive frequency, the currents through each coil are approximately in phase and reach a peak value when the external field is at a minimum, forming a tight trapping potential between the loops. About the maximum of the external $B$ field, the current in the loops will have an infinitesimal positive (negative) current amplitude and the trapping radius forms at the outer (inner) ring. Averaging over a cycle of the ac field, the time-averaged trapping potential forms a ring at approximately the mean radius of the loops. Such a ring waveguide can have large trapping frequencies due to the proximity of the trapping radius to both wires.

rf-dressed potentials for atom traps have been a topic of some interest since a proposal by Zobay and Garraway in 2001 [29]. In this Rapid Communication, we have deliberately chosen parameters to remain in a regime where the atomic magnetic moment follows adiabatically the total magnetic field and remains in an undressed $m_{F}$ state. In this parameter space, the modulation frequency of the $B$ field is much less than the Larmor frequency and both are significantly larger than the spatial trapping frequencies, as is the 
case in the time-orbiting potential (TOP) trap [21]. The extension to rf-dressed potentials is readily achievable, although it does require higher drive frequencies than those presented here.

Many applications present themselves for the ring traps proposed here. A circular waveguide is ideal for use in Sagnac interferometry [17], the study of sonic black holes [30], superfluid circulation in quantum fluids $[14,15]$, and soliton propagation $[31,32]$.

We have proposed and numerically investigated magnetic ring traps that require no external wiring. The scheme circumvents problems with roughness of the trapping potential by creating a time-averaged field that is inherently smooth with respect to deviations of the current in the conductors. We have identified a mechanism for eliminating Majorana losses by applying a quadrupole $B$ field centered on the ring. Large radius $\sim 1 \mathrm{~cm}$ traps have been described that will allow for sensitive interferometric tests, due to the macroscopic area of the rings. Smaller traps on the micrometer scale are also proposed that are amenable to microfabrication and use in atom-chip devices.

The authors thank the University of Strathclyde for financial support and Alastair Sinclair, Ifan Hughes, and Charles Adams for discussions.
[1] E. A. Hinds, C. J. Vale, and M. G. Boshier, Phys. Rev. Lett. 86, 1462 (2001).

[2] E. Andersson, T. Calarco, R. Folman, M. Andersson, B. Hessmo, and J. Schmiedmayer, Phys. Rev. Lett. 88, 100401 (2002).

[3] R. Folman, P. Krüger, J. Schmiedmayer, J. Denschalg, and C. Henkel, Adv. At., Mol., Opt. Phys. 48, 263 (2002).

[4] J. Fortágh and C. Zimmerman, Rev. Mod. Phys. 79, 235 (2007).

[5] E. A. Hinds and I. G. Hughes, J. Phys. D 32, R119 (1999).

[6] M. Trinker, S. Groth, S. Haslinger, S. Manz, T. Betz, I. BarJoseph, T. Schumm, and J. Schmiedmayer, e-print arXiv:0801.3351.

[7] W. Hänsel, J. Reichel, P. Hommelhoff, and T. W. Hansch, Phys. Rev. Lett. 86, 608 (2001).

[8] T. Schumm, S. Hofferberth, L. M. Andersson, S. Wildermuth, S. Groth, I. Bar-Joseph, J. Schmiedmayer, and P. Krüger, Nat. Phys. 1, 57 (2005).

[9] S. Kraft, A. Günther, H. Ott, D. Wharam, C. Zimmermann, and J. Fortágh, J. Phys. B 35, L469 (2002).

[10] A. E. Leanhardt, A. P. Chikkatur, D. Kielpinski, Y. Shin, T. L. Gustavson, W. Ketterle, and D. E. Pritchard, Phys. Rev. Lett. 89, 040401 (2002).

[11] A. E. Leanhardt, Y. Shin, A. P. Chikkatur, D. Kielpinski, W. Ketterle, and D. E. Pritchard, Phys. Rev. Lett. 90, 100404 (2003).

[12] M. P. A. Jones, C. J. Vale, D. Sahagun, B. V. Hall, and E. A. Hinds, Phys. Rev. Lett. 91, 080401 (2003).

[13] J.-B. Trebbia, C. L. Garrido Alzar, R. Cornelussen, C. I. Westbrook, and I. Bouchoule, Phys. Rev. Lett. 98, 263201 (2007).

[14] E. Nugent, D. McPeake, and J. F. McCann, Phys. Rev. A 68 , 063606 (2003).

[15] C. Ryu, M. F. Andersen, P. Cladé, V. Natarajan, K. Helmerson, and W. D. Phillips, Phys. Rev. Lett. 99, 260401 (2007).
[16] J. A. Sauer, M. D. Barrett, and M. S. Chapman, Phys. Rev. Lett. 87, 270401 (2001).

[17] A. S. Arnold, C. S. Garvie, and E. Riis, Phys. Rev. A 73, 041606(R) (2006).

[18] A. S. Arnold, J. Phys. B 37, L29 (2004).

[19] S. Gupta, K. W. Murch, K. L. Moore, T. P. Purdy, and D. M. Stamper-Kurn, Phys. Rev. Lett. 95, 143201 (2005).

[20] S. Wu, W. Rooijakkers, P. Striehl, and M. Prentiss, Phys. Rev. A 70, 013409 (2004).

[21] W. Petrich, M. Anderson, J. Ensher, and E. Cornell, Phys. Rev. Lett. 74, 3352 (1995).

[22] R. H. Good, Eur. J. Phys. 22, 119 (2001).

[23] At a frequency of $30 \mathrm{kHz}$, the skin depth of gold is $\delta=450 \mu \mathrm{m} \sim r_{\text {wire }}$

[24] J. D. Jackson, Classical Electrodynamics, 3rd ed. (Wiley, New York, 1999).

[25] W. Ketterle, D. S. Durfee, and D. M. Stamper-Kurn, in Making, Probing and Understanding Bose-Einstein Condensates, Proceedings of the International School of Physics "Enrico Fermi," edited by M. Inguscio, S. Stringari, and C. Wieman (IOS, Amsterdam, 1999).

[26] C. S. Adams and E. Riis, Prog. Quantum Electron. 21, 1 (1997).

[27] J. Reichel, W. Hänsel, and T. W. Hänsch, Phys. Rev. Lett. 83, 3398 (1999).

[28] A. Grabowskia and T. Pfau, Eur. Phys. J. D 22, 347 (2003).

[29] O. Zobay and B. M. Garraway, Phys. Rev. Lett. 86, 1195 (2001).

[30] L. J. Garay, J. R. Anglin, J. I. Cirac, and P. Zoller, Phys. Rev. Lett. 85, 4643 (2000).

[31] K. Kärkkäinen, A. D. Jackson, and G. M. Kavoulakis, e-print arXiv:0801.2364.

[32] N. G. Parker, A. M. Parker, S. L. Cornish, and C. S. Adams, J. Phys. B 41, 045303 (2008). 